\title{
Des espaces d'ambiguïté : les camps de réfugiés palestiniens en Jordanie
}

\author{
Luigi Achilli et Lucas Oesch ${ }^{1}$
}

es études sur les camps de réfugiés tendent à les catégoriser avant tout, soit en
tant qu'espaces extraterritoriaux d'exclusion, soit en tant que lieux du radica-

lisme et de la résistance politique (Agier 2008)². Cependant, dans cet article, en suivant l'argumentation qui est développée dans l'introduction de ce volume, nous allons montrer que l'étude des camps de réfugiés palestiniens en Jordanie conduit à une analyse plus nuancée, qui s'écarte de ces deux cas de figure. Afin de rendre compte de la multidimensionnalité qui caractérise l'expérience des réfugiés dans les camps, il est nécessaire de s'intéresser aux processus complexes à travers lesquels les camps deviennent des lieux « exceptionnels ", mais aussi " ordinaires ». Ainsi, le fonctionnement des camps en tant qu' " espaces d'ambiguïté » est le concept qui permet le mieux de saisir leurs caractéristiques. Ils sont, par exemple, à la fois des quartiers intégrés aux villes et des enclaves urbaines (Al-Husseini 2011 ; Destremau 1996; Oesch 2015a, 2015b), des lieux de revendications et de désengagement politique (Achilli 2014, 2015), etc. L'argument de cet article est donc que, en Jordanie, les camps de réfugiés ne correspondent pas au concept d' " espace d'exception » inspiré par les travaux de Giorgio Agamben $(1997$; 2003), qui fonctionnerait en permettant au pouvoir souverain de garantir une stabilité en excluant de toute participation politique la population contenue dans cet espace, et en écartant de ce fait les menaces pesant sur "l'ordre national des choses " (Malkki 1995). Au Royaume hachémite, c'est le caractère ambigu des camps de réfugiés palestiniens qui permet de garantir cette stabilité.

Comment la stabilité politique en est-elle venue à être assurée par l'ambiguïté plutôt que par la seule exception ? Cela s'explique par plusieurs facteurs. En premier lieu, historiquement, les premiers camps établis en Jordanie dans les années 50 n'ont pas été installés en vue de tenir à l'écart une menace représentée par la population palestinienne réfugiée, mais en prévision d'une

\footnotetext{
1 Les auteurs ont contribué de façon égale au texte. Les noms sont indiqués par ordre alphabétique. Nous tenons à remercier Véronique Bontemps de ses commentaires sur notre texte.

2 Toutes les citations en anglais ont été traduites en français.
} 
certaine intégration de ces réfugiés qui rapidement sont devenus de nouveaux citoyens jordaniens. Les camps ont ainsi dû être inclus dans le processus de nation building, ou du moins de state building (Milton-Edwards et Hinchcliffe 2001 : xi). En deuxième lieu, les camps répondent à des objectifs développementalistes et économiques pour diverses raisons qui ont changé avec le temps. Ils ont, par le passé, représenté une source de main-d'œuvre à proximité des centres urbains en construction (Destremau 1996 : 542), et constituent, jusqu'à nos jours, une source de financement provenant de l'aide internationale. En troisième lieu, le maintien des camps répond à des considérations géopolitiques, en fonction desquelles, à la fois les

18 Jordano-Palestiniens et les Transjordaniens, pour différentes raisons, sont opposés à tout démantèlement des camps 3 . Ces lieux sont des témoins du "droit au retour " pour les premiers, et des symboles que « la Jordanie n'est pas la Palestine " pour les seconds. En quatrième lieu, le gouvernement a fait preuve d'une volonté d'amélioration de la marginalité socio-économique des habitants des camps, et donc d'une certaine intégration de ceux-ci, afin d'empêcher la transformation de ces espaces en réservoir d'extrémistes et de groupes politiques radicaux.

Dans ce cadre, la création d'espaces d'exception et d'exclusion, ou au contraire d'espaces complémentement intégrés aurait remis en cause une partie ou la totalité de ces différents objectifs, et aurait pu s'avérer potentiellement déstabilisatrice. C'est ainsi par l'établissement d'espaces ambigus de différenciation (Oesch 2015a) que le gouvernement jordanien et la communauté internationale entendent garantir la stabilité politique et la reproduction de l'ordre " national » des choses dans le pays.

Afin de mettre en évidence cette ambiguïté, après avoir étudié son origine et sa mise en place, cet article va analyser sa spatialisation dans les camps, en

3 La catégorie « Transjordanien » fait référence à la population jordanienne identifiée ou qui s'identifie comme étant originaire des limites territoriales de l'État jordanien avant l'expansion géographique et démographique de 1948-1950, qui a vu l'annexion, par le royaume de Jordanie, du territoire connu sous le nom de Cisjordanie. La dénomination « JordanoPalestinien " est attribuée à la population qui est identifiée ou qui s'identifie comme étant originaire de Palestine, et qui s'est réfugiée sur la rive orientale du Jourdain (Transjordanie) à la suite de la guerre israélo-arabe de 1948, ou qui s'est déplacée de la rive occidentale du Jourdain (Cisjordanie) à la rive orientale, à la suite de la guerre israélo-arabe de 1967. s'intéressant à la gestion de ces espaces et à ses acteurs. Il va ensuite montrer que la reproduction de cette ambiguïté est également le fruit des pratiques des réfugiés eux-mêmes. Cette étude va se centrer sur deux camps de réfugiés palestiniens en Jordanie, à savoir les camps d'al-Wahdat et de Jabal al-Hussein, fondés au début des années 50, et tous deux situés non loin du centre-ville 
d'Amman ${ }^{4}$. Officiellement, ils abritent respectivement environ 50'ooo et 30'ooo réfugiés palestiniens enregistrés.

\section{Aux origines de l'ambiguïté}

En 1948, la création de l'État d'Israël a été l'occasion pour le roi Abdallah de Jordanie de réaliser ses objectifs expansionnistes (Shlaim 1988). À la suite de la guerre israélo-arabe qui a suivi la proclamation de l'État juif, le monarque a formellement annexé ce qui restait de la Palestine centrale. L’acquisition de la Cisjordanie a nécessité l'adoption d'une rhétorique nationaliste pour assurer son attachement à Amman. Cela s'est manifesté par l'idée de créer une identité nationale qui engloberait tous les citoyens du royaume élargi. L'expansion militaire et territoriale a été légitimée par la signature en 1949 d'un avenant à la loi de 1928 sur la nationalité, stipulant que " tous ceux qui, au moment de l'application de cette loi, ont leur résidence usuelle en Transjordanie ou sur le Territoire occidental administré par le Royaume hachémite de Jordanie, et qui détiennent la nationalité palestinienne, sont considérés comme ayant acquis la nationalité jordanienne et jouissent de tous les droits et obligations que les Jordaniens ont " (article 2, dans Massad 2001 : 39). En conséquence, jusqu’à la rupture des liens administratifs et juridiques de 1988 entre les deux rives du Jourdain, les réfugiés palestiniens qui avaient fui en direction du territoire administré par les Hachémites (Jordanie et Cisjordanie), de même que les Palestiniens qui étaient nés en Cisjordanie, ainsi que les descendants, ont obtenu l'égalité juridique avec tous les autres citoyens de l'État jordanien. Ceux étant également enregistrés auprès de l'UNRWA (Office de secours et de travaux des Nations unies pour les réfugiés de Palestine dans le Proche-Orient) ont bénéficié d'un double statut de citoyen-réfugié.

Les premiers camps de réfugiés palestiniens ont été édifiés dans le Royaume hachémite à la fin des années 40 et au début des années 50 dans le cadre de cette politique d'intégration. Lorsque l'Agence juive à Tel-Aviv a annoncé l'institution du Gouvernement provisoire d'Israël le 14 mai 1948, la guerre qui a suivi a entraîné des destructions massives et l'évacuation de la plupart des localités palestiniennes. Environ 320 'ooo réfugiés palestiniens se sont dirigés vers la Cisjordanie, 210 'ooo sont allés en direction de la Bande de Gaza et 180'ooo dans d'autres pays arabes. Environ 100'ooo Palestiniens ont trouvé refuge en Jordanie (Zureik 1996 : 2-12). Les réfu-

4 Les auteurs ont chacun mené plusieurs périodes d'enquêtes ethnographiques dans ces deux camps entre 2004 et 2012, soit avant, et pendant, la réalisation de leurs thèses de doctorat respectives. Ils ont effectué de l'observation ethnographique et conduit des entretiens semi-structurés avec les habitants et les responsables de la gestion de ces espaces. 
giés se sont concentrés autour de la capitale jordanienne et dans les centres urbains qui l'entourent. Ainsi, en novembre 1949, ils étaient environ 27'ooo à Amman et 10 'ooo dans les localités environnantes, 8'ooo à Salt, 26'00o dans la ville d'Irbid et ses environs, et 4 'ooo dans la région de Zarqa (LRCS 1949). Dans les villes et les villages, les Palestiniens ont trouvé refuge dans des maisons de proches, dans des institutions religieuses et dans des abris improvisés. Seul un nombre limité de réfugiés s'est installé dans deux camps établis par la communauté internationale en 1949 dans le pays : environ 17'0oo à Sukhneh (qui sera transféré fin 1949 à Karameh dans la vallée du Jourdain) et 2'ooo dans le camp de Zarqa. Cependant, les besoins croissants en nourriture, logement et services sanitaires ont entrainé l'UNRWA - qui a pris le relais de la Croix-Rouge et a été opérationnelle dès le mois de mai 1950 - à devoir augmenter le nombre de camps dans la région. Deux d'entre eux ont été construits à Amman - le camp de Jabal al-Hussein (1952) et Amman new camp (1955), appelé al-Wahdat par ses habitants - et un autre à Irbid (1951).

Afin de légitimer la revendication et le contrôle de la Cisjordanie, le gouvernement jordanien avait tout intérêt à assimiler davantage les Palestiniens dans la société jordanienne. Le démantèlement des camps était censé suivre. À cette fin, les camps n'ont pas été établis avec pour objectif principal de " discipliner » les réfugiés en tant qu'une anomalie dans l'ordre national des choses (Malkki 1995). L'objectif était plutôt de rassembler en quelques endroits les réfugiés qui ne pouvaient pas se permettre un autre logement. Cette assimilation a cependant été marquée par des tensions entre les Jordano-Palestiniens, le gouvernement jordanien et les Transjordaniens, relatives à l'annexion de la Cisjordanie par le régime hachémite, au développement inégal mené entre la Cisjordanie et la Jordanie - et privilégiant cette dernière -, de même qu'au favoritisme des Transjordaniens " de souche " au détriment des Jordano-Palestiniens (Massad 2001; Abu-Odeh 1999). Des tensions qui ont été illustrées par l'assassinat du roi Abdallah en 1951, abattu par un tireur palestinien alors qu'il assistait à la prière du vendredi au Dôme du Rocher à Jérusalem.

Par ailleurs, l'UNRWA, dont la principale fonction est de fournir des services aux réfugiés (de santé et sanitaires, éducatifs, sociaux et communautaires) a aussi eu un impact important sur la vie des réfugiés. À travers ses tâches, l'UNRWA a soustrait à l'État une partie de son rôle, et particulièrement de son " bio-pouvoir " (Elden 2006). Les techniques modernes de « bio-politique » de l'UNRWA visaient à inscrire les Palestiniens dans leur statut de "réfugié ». L'agence onusienne a ainsi mis en œuvre dans tous les camps de réfugiés palestiniens au Proche-Orient un certain nombre 
de pratiques administratives visant à contrôler les réfugiés et l'acheminement de l'aide. Julie Peteet souligne comment le contrôle approfondi des réfugiés par leur classement et leur comptabilisation, ainsi que par les pratiques de rationnement et d'examens médicaux « vise à une transformation subjective des personnes déplacées, les faisant passer de réfugiés révoltés et imprédictibles, à une population docile et récipiendaire d'aide internationale " $2005: 95)$. Cependant, ces pratiques de contrôle ont aussi eu des effets inattendus sur la subjectivité politique des habitants des camps. Afin de faciliter l'intégration dans le pays hôte, ces techniques de contrôle visaient à générer un groupe social homogène - les "réfugiés " - démuni de tous liens sociaux, historiques et politiques avec son passé. Le résultat fut cependant tout à fait différent. D’espaces qui avaient été créés afin de taire la situation politique des Palestiniens, en mettant en avant la question « humanitaire », les camps sont devenus des lieux où a émergé une culture de résistance politique.

À partir de la fin des années 6o, encouragé par la circulation transnationale des discours anticoloniaux, le développement d'une conscience nationale palestinienne qui s'est principalement cristallisée autour de la figure emblématique de l' « habitant du camp " (mukhayyamji) a commencé à contester l'agenda d’intégration du gouvernement jordanien. Suite à la guerre de 1967, de nombreux jeunes Palestiniens en exil se dirigent vers le mouvement de résistance de l'Organisation de libération de la Palestine (OLP). Pendant cette période de mobilisation de masse (1969-1982), un nouveau récit valorise les hommes et les femmes des camps en tant qu'incarnation de la résistance palestinienne et comme figures de l'héroïsme palestinien (voir Khalili 2007). Ce fut l'apogée du camp d'al-Wahdat en Jordanie et du camp de Chatila au Liban (voir l'article de Hala C. Abou-Zaki dans ce volume), ainsi que d'autres camps de réfugiés qui devinrent le cœur symbolique du nationalisme palestinien. L’appellation de " zone libérée » en vint à désigner les camps. Les " fils du camp ", les Fedayins (groupes de combattants palestiniens) étaient devenus l'épine dorsale militante et militaire du nationalisme palestinien, et le mot " mukhayyam " (camp de réfugiés) devint synonyme de " ma'askar " (camp d'entraînement militaire) (ibid. : 84). En Jordanie, la présence de l'OLP dans les camps était si profonde et puissante que le gouvernement s'est trouvé impuissant face à la plupart des activités militantes et militaires menées par les groupes de guérilla durant l’année qui a précédé la guerre civile jordanienne connue sous le nom de "Septembre noir ». Dans ce pays où la plupart des réfugiés palestiniens bénéficient des pleins droits de citoyenneté, l'émergence d'un quasi " gouvernement » palestinien parallèle à l'intérieur même du royaume a empiété sur la souveraineté de l'État jordanien. La 
conséquence de cet épisode résonne encore sur le processus de construction de l'identité nationale.

En 1970, la spirale de tensions entre les combattants de la guérilla palestinienne et l'armée jordanienne a déclenché une série d'événements qui ont finalement abouti à la tragique guerre civile. Le conflit a duré jusqu'en juillet 1971 et s'est achevé avec la destruction d'une grande partie des camps de la capitale jordanienne et l'expulsion des Fedayins du royaume (Massad 2001 ; Sayigh 1997). La confrontation n'a cependant pas vu la juxtaposition de deux groupes « ethniques " distincts. Non seulement une grande partie des Jordano-Palestiniens n'ont pas pris part à la guerre, mais une minorité non négligeable de Transjordaniens a rejoint les rebelles dans leur lutte contre la monarchie (Sayigh 1997).

Quoi qu'il en soit, la fin de la guerre civile a vu un changement radical dans la ligne idéologique officielle de la Jordanie. Sous la pression des nationalistes transjordaniens qui appelaient à distinguer la Jordanie de la Palestine, le processus de construction de l'identité nationale a commencé à mettre en avant, et de façon quasi exclusive, les aspects qui marquaient sa « nature " transjordanienne (Abu Odeh 1999 ; Alon 2007 ; Fruchter-Ronen 2008 ; Massad 2001; Mishal 1978 ; Salibi 1993). Si l'annexion de la Cisjordanie par le roi Abdallah a engendré la création d'une identité collective englobant des éléments palestiniens et transjordaniens, la guerre de Septembre noir et les événements qui l'ont suivi ont progressivement précipité un discours mettant en avant une relation hôte/invité. Ce discours a été exprimé par les termes arabes de muhajirin (émigrants) pour désigner les Palestiniens et ansar (hôtes) pour les Transjordaniens 5 .

La guerre civile entre les milices palestiniennes et le régime jordanien a sûrement été un premier tournant majeur, mais d'autres événements locaux et régionaux ont favorisé l'affaiblissement des politiques d’inclusion du régime. Le déclenchement

5 La référence à ces deux concepts de la tradition islamique a été faite pour la première fois par le roi Hussein -devenu monarque en 1952 - dans la foulée de la guerre civile. D’après Abu-Odeh, alors que l'intention du roi était d'invoquer cette distinction afin de renforcer l'unité nationale, les nationalistes transjordaniens ont réinterprété ces concepts afin de rappeler la présence temporaire des Palestiniens dans le royaume (1999: 211-212). de la première Intifada dans les territoires occupés en 1988 a, à ce titre, aussi été important. Non seulement ce soulèvement de la base palestinienne a remis en question l'occupation israélienne de la Cisjordanie, mais il a également contesté les revendications jordaniennes sur ce territoire. Le 31 juillet de la même année, le régime a annoncé officiellement son désengage- 
ment de Cisjordanie (Legrand 2009), et a « abandonné sa prétention de parler au nom des Palestiniens " (Nanes 2008 : 91).

La question de l'intégration des Palestiniens en Jordanie s'est cependant reposée après la signature des accords d'Oslo en 1993 qui ont établi un cadre pour la négociation d'un traité de paix israélo-palestinien. Dans la foulée, en 1994, le roi Hussein a signé le traité de Wadi Araba fixant la paix et une normalisation des relations entre la Jordanie et Israël. Ces accords et ce traité n’ont pas rencontré de grande opposition parmi la population jordano-palestinienne. Pourtant, ils ont eu un impact important sur celle-ci, étant donné qu'ils ont compromis les possibilités et le droit au retour des réfugiés palestiniens. Non seulement les accords d'Oslo ont signifié que le sort des Jordano-Palestiniens n'était plus un objet de préoccupation de l'OLP, vu que la question du droit au retour n'y avait pas été incluse, mais surtout, ils ont ainsi renforcé la disparition du principe d’un séjour " temporaire » des réfugiés en Jordanie (Abu-Odeh 1999 : 234-235).

Abu-Odeh (1999 : 228-229), un homme politique jordano-palestinien qui a occupé plusieurs fonctions dans le gouvernement jordanien, explique que jusqu'aux accords d'Oslo, la rationalité du provisoire avait permis, d'une part, aux nationalistes transjordaniens et au pouvoir royal de justifier diverses formes de discriminations envers les Jordano-Palestiniens, en considération de leur statut d'invité, et d'autre part, aux Jordano-Palestiniens de tolérer ces discriminations dont ils étaient victimes, dans la mesure où ils pouvaient admettre le caractère passager de ces pratiques dans l'attente d'une résolution de la question des réfugiés et du droit au retour. Dès l'instant où le caractère temporaire du séjour des réfugiés palestiniens en Jordanie a été plus fortement remis en question, le risque de polarisation a augmenté. D'un côté, les nationalistes transjordaniens ont plus que jamais craint la pérennisation du séjour des Jordano-Palestiniens dans le pays et, d'un autre côté, les Jordano-Palestiniens, voyant que ce même séjour était appelé à se prolonger, ont moins bien accepté les pratiques discriminatoires dont ils étaient victimes et ont désiré améliorer leurs conditions de vie dans le royaume (Abu-Odeh 1999 : 268-276). Cependant, Abu-Odeh (1999:269) précise aussi qu'une conception réaliste de la politique régionale était largement répandue parmi l'élite gouvernementale jordanienne qui voulait, dans une certaine mesure en tout cas, favoriser l'intégration des Jordano-Palestiniens, malgré une certaine opposition des nationalistes transjordaniens avec laquelle il a fallu composer. Les camps de réfugiés palestiniens en Jordanie n'ont ainsi pas cessé d'être des lieux ambigus, 
marqué à la fois par des logiques d’intégration, mais également de différenciation, et ceci dès leur origine.

\section{La spatialisation de l'ambiguïté}

Au vu de cette ambiguïté, comment se présente la gestion des camps de réfugiés palestiniens en Jordanie et leur spatialisation ? Il y a aujourd'hui plus de cinq millions de réfugiés palestiniens enregistrés auprès de l'UNRWA au Proche-Orient, dont plus de deux millions vivent en Jordanie, représentant environ un quart de la population totale du royaume (UNRWA 2015)6. Selon l'UNRWA, «les réfugiés

24 palestiniens sont des personnes dont le lieu de résidence usuel entre juin 1946 et mai 1948 était la Palestine, et qui ont perdu leur maison et leurs moyens de subsistance à la suite du conflit israélo-arabe de $1948 »$. Parmi ceux-ci, près de 400 'ooo vivent dans les dix camps de réfugiés que cogèrent actuellement le gouvernement jordanien et l'UNRWA, dont six sont situés dans la conurbation d'Amman-Zarqa (UNRWA 2015). La moitié des camps d’Amman sont de " 1948 " (dont al-Wahdat et Jabal al-Hussein), et l'autre moitié de " 1967 ».

Les camps ont été érigés sur des terrains privés qui étaient, et sont encore, provisoirement empruntés par le gouvernement jordanien à leurs propriétaires originels, et mis à la disposition de l'UNRWA afin d'accommoder une partie des réfugiés (Destremau 1996 : 532). Ainsi, les habitants des camps se retrouvent propriétaires de leur habitation, mais pas du terrain sur lequel elles sont bâties. Les camps ne sont pas gérés par les municipalités sur le territoire desquelles ils sont installés, mais sont sous le contrôle du Département des affaires palestiniennes (DPA) - une institution étatique jordanienne responsable en particulier des camps - ainsi que de l'UNRWA. Les camps se retrouvent donc avec un statut particulier d'espace provisoire et une gestion spécifique qui pourraient, au premier abord, laisser penser à une forme d' " état d'exception ». Mais, comme nous allons le détailler maintenant, l'hybridation d'une rationalité de gouvernement " étatique " promue par le DPA et d'une rationalité « humanitaire " soutenue par l'UNRWA les a en fait placé dans une position ambiguë entre quartiers " ordinaires " relativement bien intégrés dans le ${ }_{6}$ Cette proportion ne tissu urbain, et enclaves urbaines de l'exil palestinien (Destretient pas compte des Jordano-Palestiniens mau 1996 ; Oesch 2015a). qui ne se sont pas enregistrés comme refugiés auprès de l'UNRWA.

7 Les descendants sont également inclus.

À partir de mai 1950, l'aide humanitaire délivrée dans les camps est devenue entièrement du ressort de l'UNRWA. En 1951, un accord passé entre l'UNRWA et le gouvernement 
jordanien a défini les responsabilités de chacun : l'organisation des camps et de l'assistance devait être effectuée par l'UNRWA, alors que le rôle du gouvernement devait être limité au maintien de la loi et de l'ordre, ainsi qu'au soutien de l'agence onusienne dans l'exercice de sa mission humanitaire. À travers le temps, la répartition des tâches et les rôles de chacun ont cependant évolué (Al-Husseini 2011). Plusieurs aspects de la gestion des camps sont passés dans les mains du gouvernement (Jaber 1996 : 38). Ainsi, jusqu'en 1975, l'administration des camps et la gestion du bâti incombaient surtout à l'UNRWA. Depuis lors, l'accord entre le gouvernement jordanien et l'agence onusienne a été modifié, et ces fonctions incombent désormais au DPA (Destremau 1996 : 539). Aujourd'hui, le discours officiel de l'UNRWA souligne que l'agence ne " gouverne " pas les camps, et définit son rôle avant tout par la fourniture de " services " aux réfugiés : de santé et sanitaires, éducatifs, sociaux pour les plus démunis - dont la distribution de rations - et communautaires. Au nom de ces principes, elle intervient également dans d'autres domaines comme la rénovation de logements, ainsi que le nettoyage et la réfection de ruelles. L'UNRWA possède des centres de distribution des rations, des écoles, des centres de santé et des centres communautaires dans les camps, ainsi qu'un bureau pour le responsable des services de l'UNRWA dans chaque camp.

En ce qui concerne le gouvernement, le DPA possède une antenne dans chaque camp. Le rôle du département est de superviser les camps en coordination avec les autres acteurs étatiques et non étatiques qui agissent habituellement sur le territoire, ainsi que les institutions communautaires locales. Le DPA est notamment en charge du contrôle de l'utilisation des terrains et de l'application des réglementations sur les constructions. Il délivre, à cet égard, les permis pour les réhabilitations ou les agrandissements des logements, de même que pour les autorisations de création de commerces. Il veille aussi à l'état des infrastructures physiques et des équipements du camp (DPA 2008 : 88). Le département est décrit par certains de ses employés comme une forme de " petit gouvernement » ou de " petite municipalité » qui travaille en coordination avec le " grand gouvernement jordanien».

Mais, à côté de cette gestion officielle par le DPA, le gouvernement agit également par d'autres moyens dans les camps, par exemple par l'intermédiaire des Comités d'amélioration des services du camp (CSIC). En Jordanie, ces comités ont été créés à la fin des années 196o. Comme le précise Blandine Destremau (1996 : 536-537): 
"Les comités d'amélioration [...] étaient des structures proches d'associations de résidents, chargés d'une fonction de porte-parole et d'intermédiaire auprès $d u$ [DPA]. Depuis, ils sont passés sous le contrôle direct du gouvernement, par l'intermédiaire du [DPA] qui en nomme les membres. »

Destremau (1996 : 537) mentionne que les comités sont chargés de veiller à tout ce qui touche à l'amélioration des conditions collectives de vie dans les camps, en coordination avec les autres organismes impliqués dans la gestion de ces espaces (UNRWA, DPA, les donateurs étrangers, etc.). D’autres organisations, par exemple

26 caritatives, qui agissent dans les camps sont également rattachées d'une manière ou d'une autre au gouvernement, par exemple les comités du Zakat qui dépendent du Ministère des Awqaf et affaires islamiques (DPA 2008). Par ailleurs, le gouvernement intervient également dans les camps par la surveillance et le maintien de l'ordre, notamment à travers les postes de police qui sont situés dans les camps ou leurs alentours.

Les camps sont ainsi gouvernés par une gestion ambiguë, à la fois « humanitaire " émanant de l'UNRWA, mais aussi « étatique » et portée principalement par le DPA, mais également par d'autres institutions gouvernementales ou qui sont rattachées au gouvernement. De plus, au cours des dernières décennies, cette ambiguïté a continué à être reproduite, tout en se complexifiant. À la suite des accords d'Oslo de 1993 et du traité de Wadi Araba de 1994, les camps de réfugiés ont officiellement été inclus dans des programmes nationaux de développement urbain, sans que pour autant leur statut d'espace provisoire soit officiellement remis en question. Cette évolution s'est produite en raison du développement de ce que Al-Hamarneh (2002 : 184-186) analyse comme une approche " post-Oslo " réaliste et pragmatique, en fonction de laquelle les réfugiés ont réalisé que leur futur était, selon toute vraisemblance, en Jordanie, et font, depuis lors, tout pour que leur avenir y soit le meilleur possible. Ainsi, les camps ont pu être inclus, sans rencontrer une grande opposition, dans le Programme d'infrastructures communautaires (CIP). Ce dernier a été imaginé dans le cadre de la création plus large d'un filet social en Jordanie, dénommé Programme de productivité sociale (SPP) et adopté dans les années 90 à la suite des mesures d'ajustement structurel qui ont affecté le pays (Robins 2004 : 183). Début 1997, l'accord avec la Banque mondiale et les autres entités impliquées dans le programme a été conclu concernant le CIP. La Fondation de développement urbain et du logement (HUDC), un autre organisme du gouvernement jordanien, a été chargée de sa mise en œuvre. Dans les camps, les interventions ont évité des domaines sen- 
sibles et n'ont ainsi pas porté sur la création de nouvelles voies de circulation - pouvant être sources de fortes contestations en raison de la destruction des habitations qu'elles engendrent - pas plus que sur la rénovation des logements, ni l'accès formel à la propriété des terrains - pouvant de jure remettre en question le statut temporaire des camps. De plus, certaines infrastructures et services étaient déjà existants et reliés aux réseaux municipaux depuis plusieurs années (eaux, électricité, etc.). Les interventions dans le cadre du CIP ont donc été minimales et limitées à l'amélioration des infrastructures physiques (les réseaux de distribution d'eau, d'évacuation des eaux de pluie, les égouts et les systèmes électriques, dont l'éclairage public), ainsi qu'à l'amélioration des routes, ruelles et escaliers. Néanmoins, ce programme d'amélioration a contribué à transformer les camps en quartiers urbains " pauvres ", qui correspond à la vision des camps promue par de nombreux donateurs d'aide internationale (Bocco 2010 : 249).

De plus, au-delà de ce programme qui a officiellement inclus les camps, d'autres projets implantés dans les camps, ou leurs alentours, conduits par une variété d'acteurs, ont contribué à la création d'un dispositif d'aménagement urbain des camps, sans qu'il ne soit explicitement identifié comme tel, et ont conduit à un développement des zones urbaines où les camps sont situés (Oesch 2012, 2015b).

Ainsi, au cours des années 80, des Programmes de développement urbain (UDP) ont été mis en œuvre par le Département de développement urbain (UDD) - le précurseur de la HUDC - dans certains quartiers informels en Jordanie, la plupart situés dans les alentours des camps d'Amman, par exemple le quartier dénommé "Wahdat Est », et également habités en grande partie par des réfugiés palestiniens. Si ces programmes n'ont pas concerné les camps directement, ils ont contribué au développement urbain de leurs alentours directs.

Bien qu’ils soient situés à l'intérieur du territoire municipal, et pour certains comme Jabal al-Hussein et al-Wahdat proche du centre-ville, officiellement, les camps d'Amman ne sont pas inclus dans l'aménagement de la municipalité, que ce soit par exemple dans les masters plans de 1988 ou de 2008 (Joint Technical Team 1988 ; GAM 2008). Cependant, dans la même période que la mise en place du CIP, la Municipalité du Grand Amman (GAM) a tout de même procédé à la construction d'axes routiers dans les camps ou leurs alentours. Il s'agit, par exemple, de la construction de la route al-Urdun située le long d’une extrémité du camp de Jabal al-Hussein, et de l'élargissement de la route Sumayya traversant le camp d'al- 
Wahdat. Ces deux routes sont devenues des axes de transit important dans la ville. Ces constructions n'ont, à nouveau, pas rencontré de forte opposition de la part des réfugiés, malgré le fait qu'elles ont engendré des destructions d'habitations dans les camps. Elles ont contribué à fluidifier la circulation routière dans, et aux alentours, des camps, et ont placé encore davantage les camps dans le réseau routier de la ville, et renforcé leur connexion à cette derrière.

Par ailleurs, ces routes, comme les projets le développement des infrastructures dans les camps et leurs alentours, ont remis en cause la gestion particulière des camps, étant donné qu'ils ont impliqué l'intervention de nouveaux acteurs " ordinaires ", comme la GAM et la HUDC - bien que leurs interventions aient été coordonnées avec le DPA. Néanmoins, même si dans la pratique la gestion de l'espace des camps s'est donc quelque peu modifiée, officiellement les camps conservent tout de même une gestion spécifique, de même que leur statut officiel d'espace humanitaire et temporaire. Tout en se complexifiant davantage, la gestion et la spatialisation des camps continuent ainsi toujours d'être marquées par leur ambiguïté.

\section{Les habitants des camps dans l'ambiguïté}

Si l'ambiguïté permet d'analyser l'attitude du gouvernement jordanien et des acteurs internationaux envers les camps, elle permet également d'expliquer celle des réfugiés eux-mêmes.

Au fil des ans, la peur d'une réinstallation chez les réfugiés a progressivement laissé place à un sentiment de se sentir «à la maison " dans les camps, et même au développement d'une fierté d'habiter ces espaces. Les camps d'al-Wahdat et de Jabal al-Hussein sont ainsi devenus des lieux à préserver en tant que sanctuaires d'une " Palestinité ». La réappropriation et la politisation des deux camps par les habitants sont particulièrement visibles par la reconstitution dans l'espace de la mémoire du lieu d'origine et de l'exil. La configuration des camps reflète ainsi dans une large mesure l'expérience palestinienne du déplacement qui s'entrechoque avec l'organisation administrative. Les camps ont été conçus par les autorités et la communauté internationale afin d'accueillir une population perçue comme homogène. Leur espace a été divisé en unités d'habitation similaires, attribuées au hasard aux familles de réfugiés. Cependant, des regroupements spontanés de réfugiés en fonction de liens de parenté et d'origine sont apparus. Aujourd'hui, cette réorganisation spatiale d'al-Wihdat et de Jabal al-Hussein est toujours d'actualité. Ainsi, les différents quartiers (harat) des camps sont souvent identifiés en fonction de la 
provenance des habitants de certaines villes ou certains villages en Palestine, dont les noms sont désormais associés à chacune de ces parties (par exemple sab’awi « de Bir al-Sab’a » et ghazzawi " de Gaza »). Les noms des rues des camps sont également des symboles de la mémoire collective de leurs habitants. Par exemple, à al-Wahdat, certains rappellent les infrastructures qui ont contribué à caractériser le camp depuis son établissement : la rue du dispensaire (al-iyada), des écoles (al-madaris), des entrepôts de stockage de l'UNRWA (mu’an). La rue al-nadi est dénommée d'après son célèbre club sportif. D’autres évoquent des histoires de vie individuelles et des trajectoires personnelles de réfugiés comme la rue " al-liddawi » qui porte le nom de son premier marchand, et qui signifie également « de Lydd » (Jaber 1996 : 42).

Les camps sont ainsi devenus à la fois des espaces d'internement et des lieux familiers, une caractéristique qui a ouvert la voie à leur intégration progressive dans le tissu urbain et socio-économique d'Amman. Les premières structures d'habitation qui étaient autrefois appelées malja (abris), et qui avaient remplacé les tentes de toile, sont maintenant dénommées byut (maisons) par les habitants. Confrontés à une densité de population supérieure à certaines villes surpeuplées comme Mumbai et Kolkata en Inde, certains habitants des camps sont partis s'installer à l'extérieur, et d'autres ont transgressé les réglementations spatiales à l'intérieur des camps. Ils ont séparé les unités d'habitation originairement "indivisibles » entre les différents membres des familles. Au fil des ans, les maisons ont été vendues et achetées, et ont donc changé de propriétaires. Certaines habitations ont également été louées plus récemment à des réfugiés irakiens ou syriens, ainsi qu'aux travailleurs migrants égyptiens et à d'autres migrants disposant d'un faible revenu. Les loyers restent en général meilleurs marchés dans les camps comparativement aux autres quartiers centraux d'Amman. Après des changements successifs de locataires, certaines unités d'habitation ont perdu toute mémoire du propriétaire d'origine, et la spéculation immobilière est devenue une des marques tangibles de la dynamique de réappropriation de l'espace des camps par les réfugiés. L'expansion verticale des constructions a également atteint des niveaux imprévisibles. Il y a 15 ans, un deuxième étage sur un bâtiment n'était autorisé que dans des cas particuliers. Cependant, l'administration des camps par le DPA s'est avérée être relativement souple non seulement sur les pratiques de location, de vente et d'achat de logements, mais également à propos des violations des normes spatiales. Aujourd'hui, par exemple, la construction de deux étages supplémentaires (de moins de six mètres de hauteur) est tolérée sur des bâtiments commerciaux, de même que l'ajout d'un étage à un logement. La demande doit néanmoins passer par une voie officielle spécifique avant que l'autorisation ne 
soit délivrée. En conséquence, presque toutes les habitations dans les camps ont maintenant un deuxième étage, et même les bâtiments de trois voire quatre étages sont en augmentation.

Cette relative tolérance de la part du DPA envers la violation des normes spatiales du camp n'est pas en contradiction avec le contrôle accru exercé par l'État sur les camps. Elle est au contraire en accord avec la tentative du pouvoir hachémite d'assimiler les réfugiés palestiniens en Jordanie, tout en maintenant le statut temporaire des camps de réfugiés. En effet, le caractère informel et temporaire

30 des pratiques de régulation de l'État dans les camps a, d'une part, accéléré leur développement urbain, mais a, d'autre part, également permis la préservation du caractère provisoire d'al-Wahdat et de Jabal al-Hussein. Avec le développement des habitations, de même que l'agrandissement des voies de circulation et l'installation d'infrastructures comme les réseaux d'électricité, d'eau courante, et d'évacuation des eaux usagées, les réfugiés des camps d'Amman habitent désormais non seulement un espace temporaire d'urgence, mais également un espace aménagé qui devient un véritable lieu de vie.

Ainsi, alors qu'al-Wahdat et Jabal al-Hussein exhibent fièrement et tragiquement leur valeur symbolique d'icône d'une " Palestinité " et du nationalisme palestinien, ces témoins du droit au retour des réfugiés sont aussi des lieux socioéconomiquement et spatialement intégrés. Mais, les Palestiniens fondent toujours leurs représentations d'eux-mêmes dans l'espace des camps. Les réfugiés ont donc cherché à préserver ces espaces, et avant tout leur signification politique, tout en combattant leur marginalité socio-économique, et donc en favorisant leur intégration dans le territoire jordanien. Cela a surtout été le cas après les accords d'Oslo dans le cadre desquels les perspectives déjà ténues d'un rapatriement des réfugiés ont succombé à la realpolitik de l'OLP qui les a laissés de côté. Mais, dans ce processus d'intégration, les réfugiés se sont efforcés de continuer à défendre leur cause nationaliste.

Le souk (marché) du camp d’al-Wahdat illustre bien cette ambiguïté. Il est divisé en deux sections principales : la partie nord avec les marchandises alimentaires (suq al-khudra, signifiant le marché de légumes) et la partie sud avec les vêtements et autres articles (suq al-malabes, à savoir le marché des habits). Toute personne qui entre dans le marché pour la première fois est désorientée par le nombre de marchandises et de gens dans ces rues commerçantes. Le souk est un espace de sociabilité important. Une multitude de personnes y marche, vend, achète, joue à des jeux 
de société, discute, crie, traîne ou se dispute. Le regard est attiré de tous les côtés par de nombreux magasins, restaurants, fast-foods, stands et étalages de vendeurs de rue. Les commerces vendent pratiquement de tout, allant des éplucheurs à pommes de terre aux pantalons en velours côtelé, de la lingerie féminine aux petits encadrements où figurent les hadiths du Prophète ou le mot " Allah ». La plupart des fruits et légumes viennent de la région du Ghor dans la vallée du Jourdain, et les vêtements sont en provenance de Chine. Les produits tels que l'huile et la farine sont connus pour être des surplus alimentaires expédiés depuis les États-Unis. Certains vendeurs rivalisent pour crier le prix de leurs marchandises le plus fort possible, tandis que d'autres s'assoient sur des chaises en plastique et regardent passivement les passants. Une importante partie du marché est couverte. Au milieu des étalages, il y a aussi un supermarché moderne. Et parmi les petits magasins, on trouve des boutiques plus luxueuses qui rappellent des surfaces de vente telles que H\&M ou Zara. Dans ce labyrinthe en effervescence, des animaux tels que des lapins, des poulets et même des agneaux et des moutons sont exposés pour la vente.

Au moment de notre recherche, il y avait non seulement des Palestiniens qui fréquentaient le marché, mais aussi des réfugiés irakiens, des " Transjordaniens " (urdunniyyin), des Roms (nawari) et même des travailleurs domestiques philippins (filibinyin). Tous y venaient pour les prix avantageux proposés, pour l'important assortiment offert, et pour des aliments supposément plus goûteux qu'ailleurs. Cette grande variété de marchandises est une dimension de la vie du camp qui est particulièrement apprécié par ses habitants. Ces derniers soulignent souvent les avantages de vivre dans des zones densément peuplées comme al-Wahdat ou Jabal alHussein, où le coût de la vie est raisonnable et où le souk, avec ses nombreux services (khadamat), est à proximité. Ils affirment avec fierté que les produits vendus dans le souk rivalisent, voire supplantent, ceux vendus dans les zones plus chics et aisées d'Amman. Le souk des camps est ainsi un symbole à la fois de l'intégration en Jordanie des réfugiés palestiniens et de leur capacité à lutter contre l'adversité de la vie en exil en se sortant des conditions précaires auxquelles ils doivent faire face.

À ce propos, lors de nos visites dans les camps d'al-Wahdat et de Jabal al-Hussein, les employés des institutions en charge des camps ont à plusieurs reprises déclaré que les camps étaient pareils aux autres quartiers de la capitale jordanienne. Par exemple, un employé de l'UNRWA à al-Wahdat, lui-même un réfugié qui a vécu dans le camp, a répondu à la question de savoir s’il y avait des différences entre le camp et le reste de la ville que : « Non, il n’y a pas de différences, le camp est comme le balad, 
un balad palestinien !» Par ces mots, l'homme dressait un parallèle entre le souk - la zone commerciale du camp - et la vieille ville d'Amman, qui est communément dénommée balad, et qui est un espace commercial important où il existe aussi un marché couvert. Mais, en arabe, le terme de balad désigne également le " village ", la " patrie » et le « lieu d’origine ». En déclarant qu'al-Wihdat était seulement un autre «balad ", il faisait indirectement allusion au fait que le camp était devenu un centre-ville alternatif à Amman : le centre d'un espace palestinien et un symbole de la Palestine elle-même. L'association d'al-Wihdat avec le balad d'Amman est ainsi une expression de la nature ambiguë des camps de réfugiés palestiniens en Jordanie : des espaces à la fois d'intégration et d'une certaine " Palestinité ».

\section{Conclusion}

À première vue, la conception du camp en tant qu'un espace d'exception mis en place afin de garantir la souveraineté de l'État-nation en privant ses habitants de leurs droits semble trouver son application dans les camps de réfugiés palestiniens en Jordanie. Les camps possèdent un statut particulier d'espace temporaire et humanitaire prévu pour une population exilée en attente d'un retour, et ont officiellement une gestion spécifique de leur espace. Une telle approche ne tient cependant pas suffisamment compte de la nature complexe et ambiguë des camps comme alWihdat et Jabal al-Hussein à Amman : des espaces qui sont encore provisoires 60 ans après leur création, mais qui sont devenus presque impossibles à distinguer du reste de la ville au milieu de laquelle ils sont érigés, et qui sont habités par des personnes qui sont encore réfugiés après quatre générations, mais qui possèdent également les pleins droits de citoyenneté jordanienne. Ces dimensions a priori opposées et inconciliables convergent néanmoins dans ce que l'on a appelé un « espace d’ambiguïté ». Il s'agit ainsi du concept qui permet le mieux de saisir les caractéristiques des camps en Jordanie.

Ces deux camps du centre-ville d'Amman sont particulièrement emblématiques de cette nature ambiguë des camps en Jordanie, qui ne sont ni des espaces d'exception ni des espaces complètement " ordinaires ». Particulièrement à partir des accords d'Oslo, le gouvernement jordanien, soutenu par la communauté internationale, a déployé un éventail de pratiques visant le renforcement de l'assimilation et du contrôle des camps et de leurs habitants : les postes de police ont été agrandis, la supervision de l'activisme politique a été accrue, des routes traversant ou longeant les camps ont été élargies avec pour effet de renforcer l'intégration des camps à la ville, mais aussi les possibilités de surveillance, et ces espaces ont même été inclus 
dans certaines politiques de développement urbain à l'échelle nationale. Le gouvernement a aussi maintenu une attitude souple envers les infractions continues des normes spatiales par les habitants des camps, ces derniers continuant à façonner ces espaces à la fois comme des symboles d'une « Palestinité », mais aussi comme des lieux qui s'intègrent dans la capitale jordanienne. Bien que l'ensemble de ces interventions et pratiques a de facto remis en cause encore plus le caractère exceptionnel du camp, les autorités jordaniennes ont cependant affirmé à plusieurs reprises que toutes ces mesures ne visaient pas formellement à une inclusion permanente des camps sur le territoire national, pas plus qu'à la planification urbaine de la municipalité d'Amman.

Cette position ambiguë a permis de servir les intérêts du régime jordanien de plusieurs façons : en rappelant à Israël que la Jordanie ne doit pas être perçue comme une patrie alternative pour les Palestiniens ; en garantissant une rentrée d'aide internationale dirigée vers les camps; et en augmentant la probabilité d'une éventuelle indemnisation pour une réinstallation des réfugiés à l'intérieur des frontières jordaniennes. De plus, à la suite de Septembre noir, cette stratégie a également permis d'empêcher la création d'espaces complètement exclus et marginalisés échappant au contrôle de l'Etat et pouvant mettre en danger la stabilité du pays par la création de réservoirs de populations subversives. Ceci, tout en prévenant une intégration complète des habitants des camps pouvant provoquer un important mécontentement parmi les nationalistes transjordaniens dont la monarchie essaie de continuellement s'assurer le soutien (Baylouny 2008). 


\section{Références}

ABU-ODEH Adnan (1999), Jordanians, Palestinians and the Hashemite Kingdom in the Middle East Peace Process, Washington (DC), United States Institute of Peace Press. ACHILLI Luigi (2014), « Disengagement from Politics : Nationalism, Political Identity, and the Everyday in a Palestinian Refugee Camp in Jordan ", Critique of Anthropology, vol. 34, $\mathrm{N}^{\circ}$ 2, pp. 234-257.

ACHILLI Luigi (2015), « Becoming a Man in al-Wihdat: Masculine Performances in a Palestinian Refugee Camp in Jordan ", International Journal of Middle East Studies, vol $47, \mathrm{~N}^{\circ} 2$, pp. 263-280.

34 ALON Yoav (2007), The Making ofJordan: Tribes, Colonialism and the Modern State, London, IB Tauris.

AGAMBEN Giorgio (1997), Homo sacer I : Le pouvoir souverain et la vie nue, Paris, Seuil. AGAMBEN Giorgio (2003), Homo Sacer II : État d'exception, Paris, Seuil.

AGIER Michel (2008), Gérer les indésirables : des camps de réfugiés au gouvernement humanitaire, Paris, Flammarion.

AL-HAMARNEH Ala (2002), "The Social and Political Effect of Transformation Processes in Palestinian Refugee Camps in the Amman Metropolitan Area (1989-99) ", in Jordan in Transition 1990-2000, G. Joffe (dir.), London, Urst \& Company.

AL-HUSSEINI Jalal (2011), " The Evolution of the Palestinian between Logics of Exclusion and Integration ", in Villes, pratiques urbaines et construction nationale en Jordanie, M. Ababsa et R. Daher (dir.), Beirut, Presses de l'IFPO.

BAYLOUNY Anne-Marie (2008), “ Militarizing Welfare : Neo-liberalism and Jordanian Policy ", Middle East Journal, vol. 62, $\mathrm{N}^{\circ}$ 2, pp. 277-303.

BOCCO Riccardo (2010), "UNRWA and the Palestinian Refugees : A History within History ", Refugee Survey Quarterly, vol. 28, N²-3, pp. 229-252.

DESTREMAU Blandine (1996), "Les camps de réfugiés palestiniens et la ville : entre enclave et quartier » in Amman : ville et société(the city and its society), J. Hannoyer et S. Shami (dir.), Beyrouth, CERMOC.

DPA (2008), 60 Years Serving Refugee Camps, Amman, Department of Palestinian Affairs, The Hashemite Kingdom of Jordan.

ELDEN Stuart (2006), "Spaces of Humanitarian Exception ", Geografiska Annaler: Series B, Human Geography, vol. 88, N ${ }^{\circ}$, pp. 477-485.

FRUCHTER-RONEN Iris (2008), « Black September : The 1970-71 events and their impact on the formation of Jordanian national identity ", Civil Wars, vol. 10, pp. 244260.

GAM (2008), The Amman Plan : Metropolitan Growth, Amman, Greater Amman Municipality. 
JABER Hana (1996), "Le camp de al-Wihdat, entre norme et transgression ", Revue d'études palestiniennes, $\mathrm{N}^{\circ} 8, \mathrm{pp} \cdot 37-48$.

JOINT TECHNICAL TEAM (1988), Greater Amman Comprehensive Development Plan, 1. Main Report, Report 5 : Final Report, Amman, Greater Amman Municipality and Dar Al-Handasah Consultants.

KHALILI Laleh (2007), Heroes and Martyrs of Palestine: The Politics of National Commemoration, Cambridge, Cambridge University Press.

LEGRAND Vincent (2009), Prise de décision en politique étrangère et géopolitique : le triangle «Jordanie-Palestine-Israël » et la décision jordanienne de désengagement de Cisjordanie (1988), Bruxelles, P.I.E. Peter Lang.

LRCS (1949), Report to the UNRPR on the part of the League of Red Cross Societies in the Palestine refugees relief programme for the month of November, Beirut, League of Red Cross Societies.

MALKKI Liisa (1995), Purity and Exile: Violence, Memory, and National Cosmology among Hutu Refugees in Tanzania, Chicago, University of Chicago Press.

MASSAD Joseph A. (2001), Colonial Effects : The Making of National Identity in Jordan, New York, Columbia University Press.

MILTON-EDWARDS Beverley et Peter HINCHCLIFFE (2001), Jordan : A Hashemite Legacy, London, Routledge.

MISHAL Shaul (1978), Bank/East Bank: The Palestinians in Jordan, 1949-1967, New Haven, Yale University Press.

NANES Stefanie (2008), "Choice, loyalty, and the melting pot : Citizenship and national identity in Jordan ", Nationalism and Ethnic Politics, vol. 14, pp. 85-116.

OESCH Lucas (2012), " Le développement urbain des camps de réfugiés palestiniens et des quartiers informels à Amman : dispositif d'aménagement de territoires fragmentés ", Cahier du GREMAMO, N²1, pp. 155-176.

OESCH Lucas (2015a), " Le gouvernement des camps palestiniens en Jordanie à la croisée de différentes rationalités ", in Acteurs et pouvoirs dans les villes du Maghreb et du Moyen-Orient, K. Bennafla (dir.), Paris, Karthala.

OESCH Lucas (2015b), "L'amélioration des conditions de vie dans les camps de réfugiés palestiniens à Amman ou la dé-théâtralisation de l'urbanisme jordanien ", Articulo-Journal of Urban Research, $\mathrm{N}^{\circ} 7$.

PETEET Julie (2005), Landscape of Hope and Despair : Palestinian Refugee Camps, Philadelphia and Bristol, University of Pennsylvania Press.

ROBINS Philip (2004), A History of Jordan, Cambridge (UK), Cambridge University Press.

SALIBI Kamal (1993), The Modern History of Jordan, London, IB Tauris.

SAYIGH Yezid (1997), Armed Struggle and the Search for State : The Palestinian National Movement, 1949-1993, Oxford, Oxford University Press. 
SHLAIM Avi (1988), Collusion across the Jordan : King Abdullah, the Zionist Movement, and the Partition of Palestine, New York, Columbia University Press.

UNRWA (2015), UNRWA in Figures (as of 1 Janualy 2015), Jerusalem, Communications division, UNRWA Headquarters.

ZUREIK Elias (1996), Palestinian Refugees and the Peace Process, Washington D.C., Institute for Palestine Studies. 